\title{
Incorporación de técnicas para favorecer el aprendizaje activo en la asignatura "Evaluación técnico-económica de sistemas energéticos"
}

Jorge Payáa

${ }^{a}$ Instituto de Ingeniería Energética, Universitat Politècnica de València

Camino de Vera s/n Edificio 8E semisótano frente acceso J, Valencia, España

E-mail: jorge.paya@iiie.upv.es (Jorge Payá)

\begin{abstract}
The present work describes the active learning approach followed in the subject "Techno-economical assessment of energy systems". In first place, the employed techniques are described and in second place, the results are discussed from the point of view of the teacher and the students, by means of a survey which was answered by 34 of the 36 students. The results indicate that the students have a very positive perception with respect to their training when adopting techniques such as group discussion, evaluation with projects or oral presentations. A novel aspect has been including half an hour of autonomous work of the students in each practical lesson, where they had to solve on their own typical simulation errors. $73 \%$ of the students agreee that this method developed their autonomy. Globally, and although the results are very positive (97\% satisfaction with the teacher and $86 \%$ with the subject), the teaching can still be improved by further working on the teaching material (contents) aswell as by better adapting the project to the contents and competences which are developed through the subject.
\end{abstract}

Keywords: project-based learning, transversal competences, evaluation, active learning

\footnotetext{
Resumen

El presente trabajo describe la metodología de aprendizaje activo adoptada en la asignatura de "Evaluación técnico-económica de sistemas energéticos". En primer lugar se describen las distintas técnicas empleadas en clase y en segundo lugar se comenta su utilidad tanto desde el punto de vista del profesor como de los alumnos, a través de un sondeo al que contestaron 34 de los 36 alumnos matriculados. Los resultados muestran que los estudiantes perciben de manera muy positiva en su formación técnicas como presentaciones orales de los alumnos, evaluación por proyectos o discusión en grupos. Un aspecto novedoso ha sido incluir en las prácticas media hora de trabajo autónomo de los alumnos para detectar problemas típicos de simulación. $73 \%$ de los alumnos indican que esto aumentó su autonomía. Globalmente, aunque los resultados son muy buenos $197 \%$ de
} 
satisfacción con el profesor, 86\% con la asignatura en general), todavia hay margen de mejora sobre todo en los contenidos y en el enfoque del proyecto, para que sea más acorde con los conceptos y competencias trabajados en la asignatura.

Palabras clave: evaluación por proyectos, competencias transversales, aprendizaje activo

\section{Introducción}

La tendencia actual en la educación es enseñar al alumno a que aprenda, dejando atrás metodologías más pasivas como las clases magistrales donde la interacción con el alumno era muy reducida. También se está tendiendo hacia un sistema de enseñanza-aprendizaje basado en competencias (Tardif, 2006), lo cual abre preguntas importantes acerca de su posible evaluación (Castro, 2011, Fernandez, 2010, Villa, 2011).

Con el objetivo de favorecer el aprendizaje activo de los alumnos, una parte esencial consiste en variar las técnicas empleadas. Esto rompe la rutina o parsimonia de parte del alumnado, que de otra manera estaría más distraído, y favorece una mayor atención.

El presente trabajo describe las metodologías implantadas así como los resultados obtenidos, tanto desde el punto de vista de los alumnos como del profesor. La metodología adoptada es una continuación del trabajo publicado recientemente (Payá, 2014).

\section{Objetivos}

El trabajo realizado se enmarca dentro del Máster Universitario en Tecnología Energética para el Desarrollo Sostenible impartido en la Universitat Politècnica de València (UPV). En concreto, en la asignatura de "Evaluación técnico-económica de sistemas energéticos" que se imparte íntegramente en inglés en la Escuela Técnica Superior de Ingenieros Industriales (ETSII).

\section{Desarrollo de la innovación}

\subsection{Metodología}

Las clases se han enfocado con el objetivo de favorecer el aprendizaje activo de los alumnos. Para ello, se han combinado distintas técnicas tales como:

- Preguntas frecuentes a los alumnos durante las clases

- Discusiones en grupo

- Ejercicios

- Prácticas

- Resolución de problemas en prácticas informáticas

- Proyecto final 
Se han utilizado como herramientas, dentro de la aplicación de "PoliformaT" disponible en la UPV, los recursos así como el espacio compartido entre alumnos y profesor.

El sistema de evaluación escogido y la forma en el que se adapte a los contenidos y competencias que se trabajan en clase es un aspecto fundamental. En la presente asignatura, la evaluación se realiza mediante los siguientes pesos:

- $40 \%$ examen escrito de respuesta abierta

- $20 \%$ prácticas informáticas (6 de dos horas cada una)

- $40 \%$ proyecto en parejas evaluado mediante una presentación oral

\section{Resultados}

A continuación, se comentan los resultados más relevantes de la aplicación de la metodología anterior, basándose en un sondeo que se realizó a los alumnos, y al que contestaron 34 de los 36 alumnos matriculados.

\subsection{Evaluación}
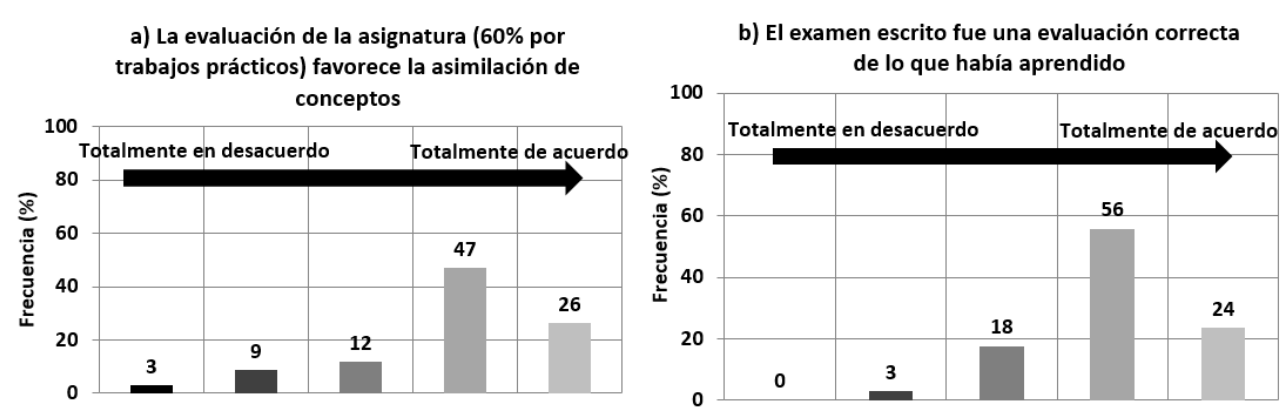

c) El proyecto final de la asignatura es una manera buena de trabajar los conceptos y competencias que debería alcanzar en la asignatura

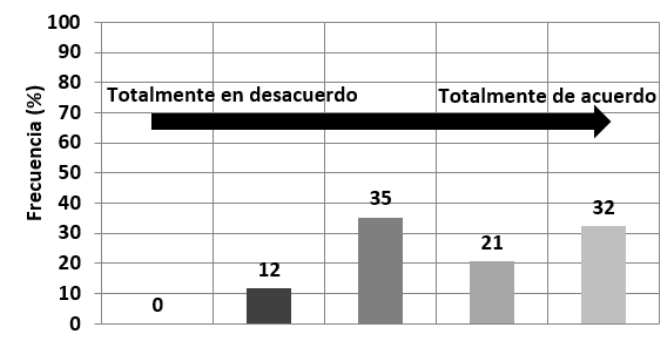

Fig. 1 Resultados de la evaluación de la asignatura

En la Fig. 1 se ha resumido la opinión del alumnado respecto a la evaluación de la asignatura. Cabe destacar que el hecho de evaluar con un peso del $60 \%$ los trabajos de tipo práctico (tanto las prácticas informáticas como el proyecto lo son) parece favorecer la asimilación de conceptos (Fig. 1a) para un $73 \%$ de los alumnos. El examen escrito, según $80 \%$ de los alumnos, evaluó correctamente lo que habían aprendido los alumnos. Esta retro- 
alimentación de los alumnos es importante para garantizar que se les evalúa acorde a lo que se les enseña en clase.

Un 53\% de los alumnos están de acuerdo en que el proyecto final es una buena manera de trabajar los conceptos y competencias que deberían adquirir en la asignatura (Fig. 1c). Sin embargo, 35\% seleccionaron el término medio y un $12 \%$ estaban parcialmente en desacuerdo, luego la opinión respecto a los proyectos fue bastante dispersa. En la sección 4.3 de balance general se comenta qué aspectos se podrían mejorar tanto en el proyecto como en el resto de la asignatura.

\subsection{Desarrollo de competencias}

En general, tal y como muestra la Fig. 2, las distintas técnicas empleadas favorecieron con éxito el desarrollo de competencias transversales. Por ejemplo, $79 \%$ de los alumnos consideran que las presentaciones fueron útiles para desarrollar habilidades de comunicación oral (Fig. 2a). Conviene señalar que algo que los alumnos valoran muy positivamente es que tras concluír las presentaciones, el profesor destaca aspectos positivos de las presentaciones, así como puntos a mejorar de las mismas. En caso contrario, si los alumnos no tuvieran otra retro-alimentación más allá de la nota, seguramente las presentaciones habrían tenido un impacto menor en su proceso de aprendizaje.
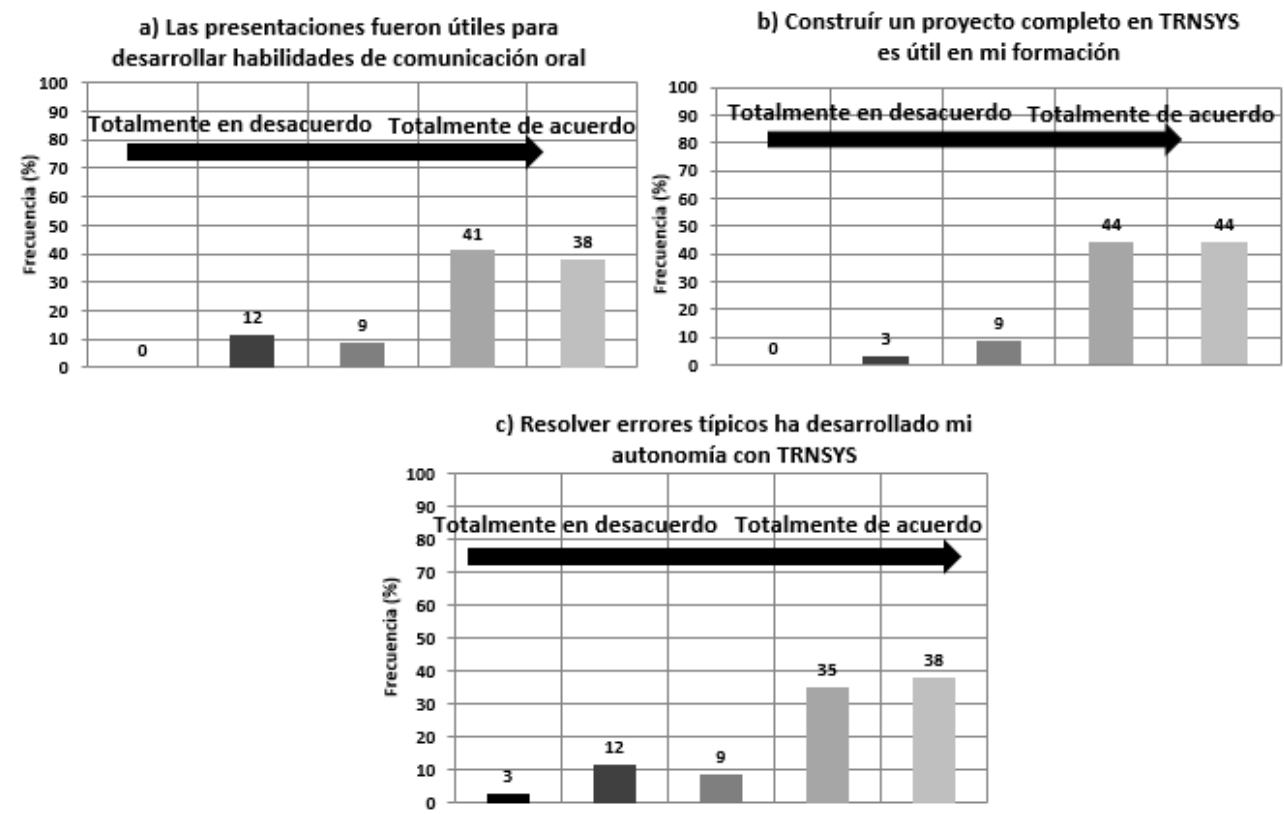

Fig. 2 Impacto de las técnicas empleadas sobre el desarrollo de competencias

En la asignatura, se trabaja con una herramienta informática llamada TRNSYS para la simulación de sistemas energéticos en régimen transitorio. Se trata de una herramienta compleja, por lo que, además de utilizarse en 6 prácticas, se dedicó una clase entera para que los alumnos construyeran un proyecto completo empezando desde cero, tal y como

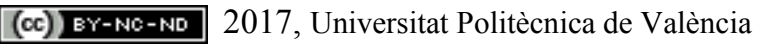


harían si tuvieran que afrontar un problema sólos por su cuenta. Un $88 \%$ de los alumnos están de acuerdo en que este proyecto fue útil en su formación (Fig. 2b).

Finalmente, conviene señalar que en todas las prácticas, se dedicó la última media hora para que los alumnos resolvieran errores que aparecían en distintos proyectos que les entregaba el profesor. Todos ellos contenían los errores típicos que suelen cometer los alumnos. Los resultados fueron muy bien acogidos, pues un $73 \%$ de los alumnos están de acuerdo con que este enfoque desarrolló su autonomía trabajando con TRNSYS (Fig. 2c). Aunque el sondeo no lo preguntaba de forma explícita, esto permitió trabajar tanto su autonomía como su sentido crítico, pues en lugar de partir de que las simulaciones eran todas correctas, partían asumiendo que contenían un error, y muchos tenían una gran motivación por encontrarlo.

\subsection{Balance general}

Como balance general de las distintas técnicas empleadas, se preguntó a los alumnos acerca de la utilidad de cada una de ellas (Fig. 3). Tanto la discusión en grupos acerca de preguntas abiertas, como realizar preguntas continuamente en las clases fue apreciado positivamente. Además, $91 \%$ de los alumnos opinan que es esencial acompañar las clases de teoría con ejercicios para asimilar mejor los conceptos.
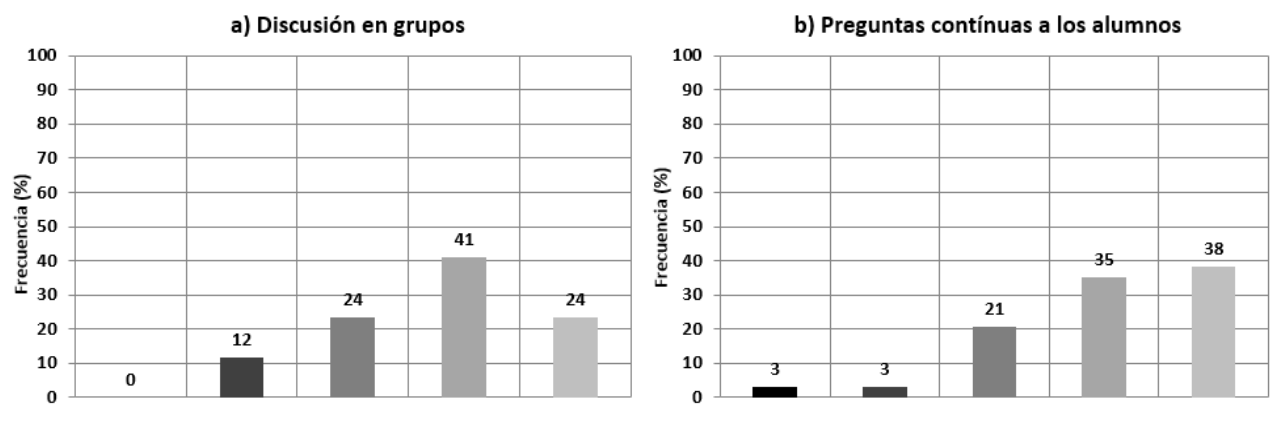

c) Proyecto final
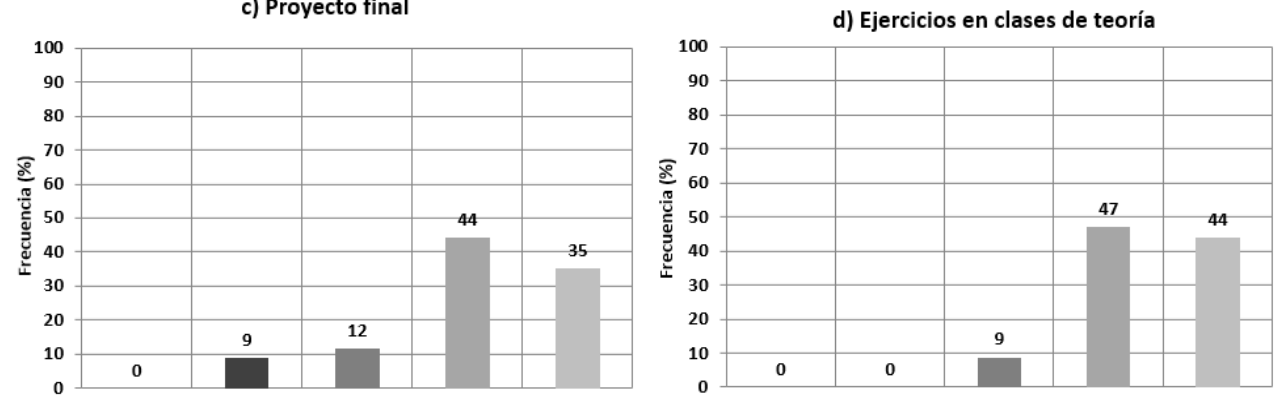

Fig. 3 Utilidad de distintas técnicas empleadas desde el punto de vista del alumnado

Curiosamente, en la pregunta 3c) los alumnos contestaron en un 79\% que les parecía positivo en su formación realizar un proyecto final. Sin embargo, sólo un $53 \%$ de los alumnos están de acuerdo en que el proyecto final es una buena manera de trabajar los 
conceptos y competencias que deberían adquirir en la asignatura (Fig. 1c). Esta diferencia parace deberse a que la realización de un proyecto les hace trabajar competencias como la autonomía, la capacidad de autocrítica, la búsqueda y selección de información, y como sistema de evaluación en sí, parece ser muy positivo. Sin embargo, a la hora de llevarlo a la práctica, quizás los alumnos no tuvieron suficiente tiempo para realizar los proyectos, y deberían mejorarse en años posteriores los conceptos y competencias que se trabajan en los mismos.

A nivel global, en la Fig. 4 se muestra el nivel de satisfacción general respecto al profesor, y respecto a la asignatura en sí. Pese a que la encuesta es anónima, probablemente haya alumnos que no contesten libremente a esta pregunta, lo cual tendería a favorecer los resultados de las encuestas. En todo caso, en las respuestas al sondeo, un $97 \%$ de los alumnos estaban satisfechos positivamente con el profesor, lo cual parece responder a que perciben positivamente la actitud dinámica del profesor y su voluntad de mejorar continuamente. Un 15\% de los alumnos seleccionaron el término medio respecto al nivel de satisfacción de la asignatura, lo cual pese a ser una cifra baja, parece indicar que a nivel de asignatura, todavía hay margen para mejorar el material que se entrega a los alumnos, añadiendo algún ejercicio adicional y renfocando ligeramente el proyecto final.
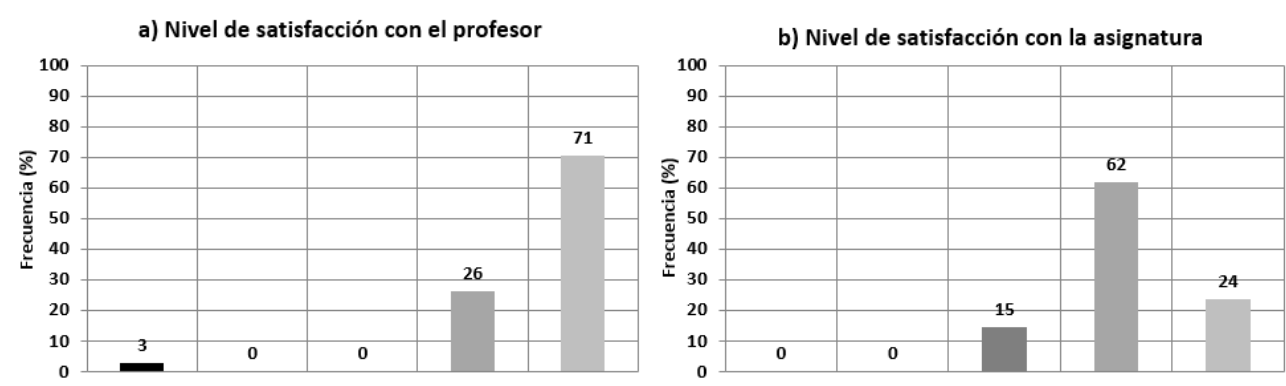

Fig. 4 Desarrollo de la competencia transversal de comunicación oral

La nota media de la asignatura en el curso 2016-2017 fue de 7.8, frente a un 8.7 en el curso anterior. Sin embargo, no se puede deducir una relación directa con la metodología adoptada, pues las diferencias son pequeñas y los factores muy diversos, ya que hubo dos profesores distintos en el curso anterior y con menos alumnos en dicho curso (25) frente a los 35 del curso 2016-2017. En las encuestas oficiales realizadas al alumnado, el profesor recibió una nota media de 9.57 frente al 8.23 del curso anterior, lo cual parece indicar la buena repercusión que ha tenido la metodología adoptada sobre la opinión de los alumnos.

\section{Conclusiones}

En el presente trabajo se ha presentado la metodología planteada dentro de la asignatura de "Evaluación técnico-económica de sistemas energéticos". Se han incorporado en las clases una gran variedad de técnicas con el objetivo de favorecer el aprendizaje activo de los

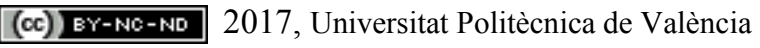


alumnos, los cuales han valorado muy positivamente todas ellas. Entre las metodologías más apreciadas por los alumnos, cabe destacar la introducción sistemática de ejercicios en clases de teoría, la discusión entre grupos, las preguntas contínuas a los alumnos, y la resolución de errores típicos por parte de los alumnos en la última media hora de las prácticas.

Pese a que un $86 \%$ de los alumnos han valorado positivamente la asignatura, las encuestas muestran que hay margen de mejora, sobre todo en lo referente al material que se les entrega, mejorando contenidos, por ejemplo introduciendo más ejercicios, o renfocando el proyecto para que puedan desarrollarlo con más tiempo y que esté más acorde con los contenidos y competencias trabajados durante la asignatura.

\section{Referencias}

CASTRO, M. (2011). “¿Qué sabemos de la medida de las competencias? Características y problemas psicométricos en la evaluación de competencias" en Bordón, vol. 63, issue 1, p. 109-123.

FERNÁNDEZ, A. (2010). "La evaluación orientada al aprendizaje en un modelo de formación por competencias en la educación universitaria" en Revista de Docencia Universitaria, vol. 8, issue 1, p. 11-34.

PAYÁ, J., y ROYO, R. (2014). “ Experiencia de un proyecto de aprendizaje basado en proyectos prácticos: Análisis de la eficiencia energética de una vivienda" en Jornadas de Innovación Educativa $y$ Docencia en Red (In-Red 2014). Valencia : Universitat Politènica de València. 878-886.

TARDIF, J. (2006). L'évaluation des compétences. Documenter le parcours de développement. Montréal: Chenelière Education.

VILLA, A. y POBLETE, M. (2011). "Evaluación de competencias genéricas: principios, oportunidades y limitaciones" en Bordón, vol. 63, issue 1, p. 147-170. 